\title{
Fat Struts: Constructions and a Bound
}

\author{
N. J. A. Sloane*, Vinay A. Vaishampayan*, Sueli I. R. Costa ${ }^{\dagger}$ \\ *AT\&T Shannon Labs, Florham Park, NJ 07932, USA. Email: \{njas,vinay\} @ research.att.com \\ †University of Campinas, Campinas, SP 13083-970, Brazil. Email: sueli@ime.unicamp.br
}

\begin{abstract}
Given a lattice $\Lambda \subset \mathbb{R}^{n}$, a cylinder anchored at two lattice points is called a strut if its interior does not contain a lattice point. We wish to determine the maximum radius of a strut of given length. Optimal struts are constructed in $\mathbb{Z}^{3}$ and $\mathbb{Z}^{4}$. We also derive, using a nonconstructive but elementary argument, an achievable lower bound on the product $l \epsilon^{n-1}$, where $l$ and $\epsilon$ are the length and radius of a strut in $\mathbb{Z}^{n}$. The motivation for the problem comes from studying nonlinear analog communication systems.
\end{abstract}

\section{INTRODUCTION}

Given a lattice $\Lambda \subset \mathbb{R}^{n}$, a strut is an $n$-dimensional cylinder with circular cross-section whose face centers coincide with distinct lattice points and whose interior does not contain a lattice point, i.e. a strut is an unobstructed cylinder anchored by two lattice points. Our problem is to determine the maximum radius of a strut of given length. We will refer to a strut of maximal radius between two anchor points as a fat strut. It is clear that longer struts must be thinner. This is illustrated in Fig. 1 for the integer lattice $\mathbb{Z}^{2}$. The case $n=2$ can be solved explicitly. A strut anchored between two given lattice points which are distance $l$ apart has maximal radius $\epsilon=1 / l$, independent of the choice of the anchor points.

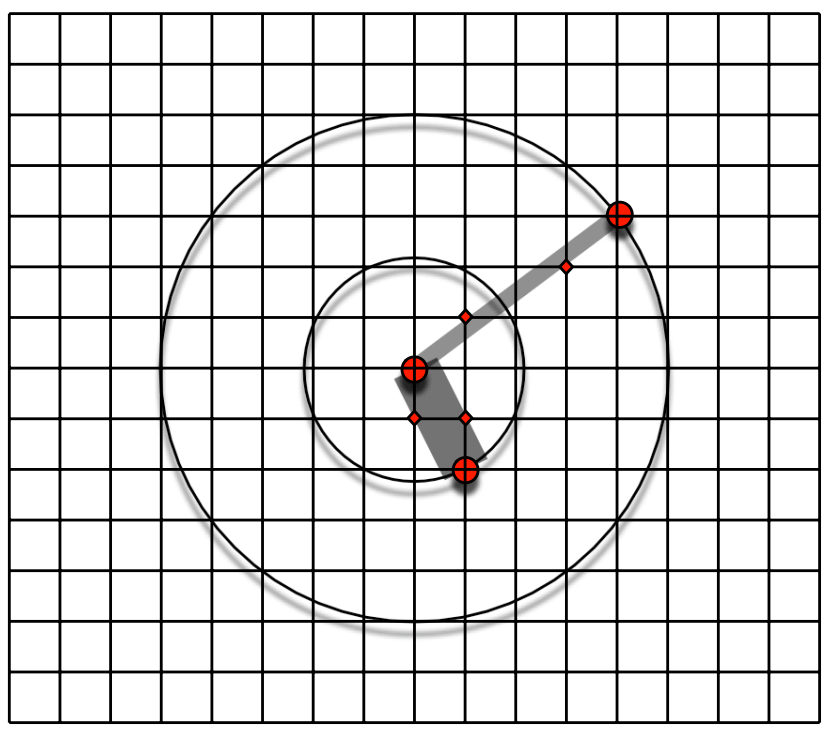

Fig. 1. Struts in the two-dimensional lattice $\mathbb{Z}^{2}$. Longer struts are thinner. Diamonds indicate lattice points that constrain the radius.

In dimensions $n>2$ the situation is different. Two fat struts of the same length may have different radii, and the question then is, for a given length, to find good endpoints for the fattest of fat struts and to determine its radius. Without loss of generality, we may fix one endpoint of the strut at the origin.

In this paper we will construct optimal sequences of struts in $\mathbb{Z}^{n}$ for $n=3,4$ and for $n>1$ we give an achievable lower bound on $l \epsilon^{n-1}$ using a nonconstructive averaging argument. The problem has its roots in communication theory [11] (see Sec. (II), but is an interesting geometric question in its own right. It is worth contrasting the problem with the result of Heppes [6] and Horvath [8] that for any lattice sphere packing in dimension three or higher there is always an infinite cylinder of nonzero radius which does not touch any of the spheres.

The paper is organized as follows: the communicationtheoretic motivation is described in Sec. [I] fundamental constraints on achievability are stated in Sec. III] optimal constructions for $n=3,4$ are given in Sec. IV] and the lower bound is derived in Sec. V

\section{Motivation: Combined Source And Channel CODING}

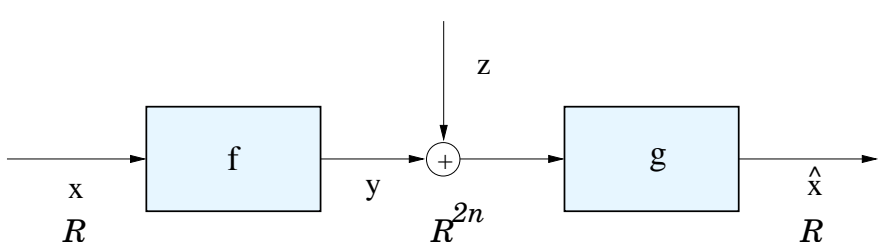

Fig. 2. Communication system where the fat strut problem arises.

The fat strut problem arises when attempting to optimize the performance of the communication system illustrated in Fig. 2 This system was studied in [10], [11], and is a model for a nonlinear analog communication system studied by Shannon [9]. The codes that we use are related to certain spherical codes which underlie cyclic group codes, studied earlier by Biglieri and Elia [1]. A continuous-alphabet random variable is to be transmitted over an independent vector Gaussian channel of dimension $2 n$. An input $x \in[-1 / 2,1 / 2]$ is mapped by an encoder $f: \mathbb{R} \rightarrow \mathbb{R}^{2 n}$ to a vector $\boldsymbol{y}=f(x)$. The mapping is given by $f(x)=\alpha\left(v_{1}(x), v_{2}(x), \ldots, v_{n}(x)\right)$, where $v_{i}(x)=(2 \pi / \sqrt{n})\left(\cos a_{i} x, \sin a_{i} x\right), i=1,2, \ldots, n$, $\alpha \in \mathbb{R}$ is a scale factor chosen to satisfy a power constraint and $\boldsymbol{a}=\left(a_{1}, a_{2}, \ldots, a_{n}\right) \in \mathbb{Z}^{n}$. The image of $[-1 / 2,1 / 2]$ under $f$ is a curve on a torus contained in a sphere in $\mathbb{R}^{2 n}$. The decoder observes a noisy version of the transmitted signal $y$ and computes $\hat{x}$, an estimate of $x$ chosen to minimize $E\left[(X-\hat{X})^{2}\right]$, the reconstruction mean squared error (mse). 
The performance of this system, general characteristics of which are illustrated in Fig. 3, is determined by two geometric parameters of the curve, the amount by which the length is increased, or its stretch, $\|\dot{f}(x)\|=(2 \pi / \sqrt{n})\|\boldsymbol{a}\|$, which in this case is independent of $x$, and its minimum distance

$$
d_{\min }(\boldsymbol{a}):=\min _{\boldsymbol{n} \in \mathbb{Z}^{n}} \min _{t}\|\boldsymbol{a} t-\boldsymbol{n}\|
$$

where the outer minimization is over vectors $\boldsymbol{n}$ which are not multiples of $\boldsymbol{a} . d_{\min }(\boldsymbol{a})$ is a measure of the minimum distance between different laps or folds of the curve. The slope of the performance characteristic above the threshold signal-to-noise ratio, $s n r_{t h}$, increases with stretch, and the value of $s n r_{t h}$ decreases as $d_{\min }(\boldsymbol{a})$ is increased. In order to optimize the

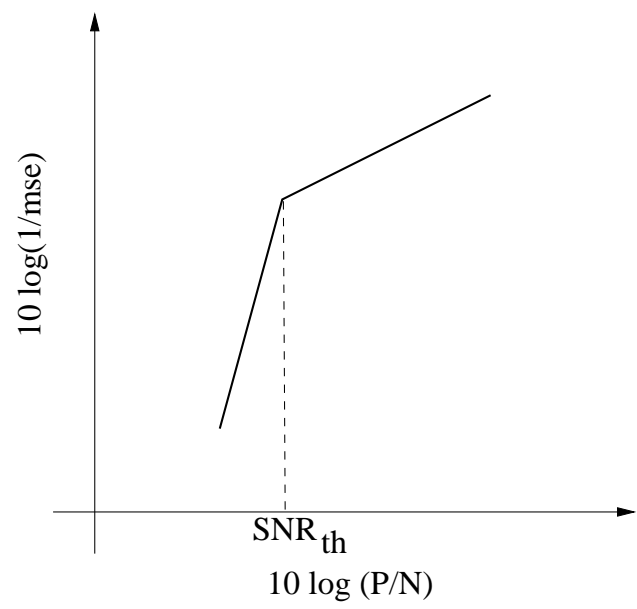

Fig. 3. An illustration of typical behavior for the kind of communication system being analyzed. $P=\alpha^{2}$ is the transmitted power and $N$ is the noise variance.

performance of this communication system, we would like to select $\boldsymbol{a}$ so that $d_{\min }(\boldsymbol{a})$ is maximized for fixed $\|\boldsymbol{a}\|$. Note that $d_{\min }(\boldsymbol{a})$ is the radius of the fat strut anchored at the origin and $\boldsymbol{a}$, while $\|\boldsymbol{a}\|$ is its length. The design problem therefore reduces to the fat strut problem.

\section{A Fundamental CONSTRAint}

The inner minimum in (1) is achieved by the value of $t$ for which $\langle\boldsymbol{n}-t \boldsymbol{a}, \boldsymbol{a}\rangle=0$ and is equal to the length of the vector obtained by projecting $\boldsymbol{n}$ into the subspace $\boldsymbol{a}^{\perp}$ orthogonal to $\boldsymbol{a}$. The radius of the fat strut anchored between the origin and $\boldsymbol{a}$ is therefore equal to the length of the shortest vector in the lattice $\Lambda_{a}$ obtained by projecting $\mathbb{Z}^{n}$ onto $a^{\perp}$. The determinant of $\Lambda_{a}$ (the square of the volume of a fundamental region) is $\operatorname{det}\left(\Lambda_{\boldsymbol{a}}\right)=1 /\|\boldsymbol{a}\|^{2}$, and its packing density is $\Delta=V_{n-1}\|\boldsymbol{a}\|\left(d_{\min }(\boldsymbol{a}) / 2\right)^{n-1}$, where $V_{n}$ is the volume of a unit sphere in $\mathbb{R}^{n}$. This tells us that the product $d_{\min }^{n-1}(\boldsymbol{a})\|\boldsymbol{a}\|$ can be made only as large as lattices in $(n-1)$-dimensional space will allow, and that our design problem can be reduced to one of selecting $\boldsymbol{a}$ such that $\Lambda_{\boldsymbol{a}}$ has the greatest possible packing density.

The densest lattice packings in dimensions $n \leq 8$ are known and are summarized in Table [] based on Table 1.2 in [3]. For

$\begin{array}{ccccccc}2 & 3 & 4 & 5 & 6 & 7 & 8 \\ A_{2} & D_{3} & D_{4} & D_{5} & E_{6} & E_{7} & E_{8} \\ 1 / \sqrt{12} & 1 / \sqrt{32} & 1 / 8 & 1 / \sqrt{128} & 1 / \sqrt{192} & 1 / 16 & 1 / 16 \\ & & & \text { TABLE I } & & & \end{array}$

DiMENSION (ROW 1), LATTICE (ROW 2), CENTER DENSITY $\Delta / V_{n}$ (ROW 3 ) FOR DENSEST LATTICE PACKINGS [3] IN DIMENSIONS $2-8$.

$n=3$ this table tells us that $\boldsymbol{a}$ should be chosen so that $\Lambda_{\boldsymbol{a}}$ is close to the hexagonal lattice $A_{2}$, which has packing density $\pi / \sqrt{12}$. Similar remarks hold for higher dimensions. However we have an additional constraint, namely that our lattices must be obtained by projecting $\mathbb{Z}^{n}$ onto one lower dimension. Thus it is not clear that the optimal packing densities stated in Table II can be achieved. We settle this question for $n=3,4$ by giving explicit constructions. We also show that there is a sequence of vectors $\boldsymbol{a} \in \mathbb{Z}^{3}$ such that $\Lambda_{\boldsymbol{a}}$ converges to any desired two-dimensional lattice.

For dimensions $n \geq 9, n \neq 24$, the optimal lattice packing densities are as yet unknown. However, in 1905, Minkowski made a conjecture that there exists a lattice $\Lambda \subset \mathbb{R}^{n}$, with packing density [3]

$$
\Delta \geq \zeta(n) / 2^{n-1}
$$

where $\zeta(n)=\sum_{k=1}^{\infty} 1 / k^{n}$ is the Riemann zeta-function. The conjecture was proved by Hlawka in 1944 [7] (see also [4]). The Minkowski-Hlawka theorem is proved by an averaging over a general class of lattices.

Our lattices are more restricted than those in the MinkowskiHlawka theorem and it is unclear how this constrains the achievable packing density. We will give an achievability result that is close to the Minkowski-Hlawka bound, using only elementary arguments.

\section{CONSTRUCTIONS FOR $n=3,4$}

In dimensions 3 and 4 we can construct families of projected lattices which approach the best possible packing densities. To determine the packing density of the projected lattice $\Lambda_{a}$ we need its determinant, $1 /\|\boldsymbol{a}\|^{2}$, and the length of a shortest vector. In low dimensions we can find the length of a shortest vector by finding a Minkowski-reduced basis (cf. [3]). We proceed by finding a Gram matrix for $\Lambda_{\boldsymbol{a}}$, Minkowski-reducing it, and showing that, up to a scale factor, this converges to a a Gram matrix for the target lattice in $\mathbb{R}^{n-1}$.

For $n=3$ we can make the projected lattice converge to the hexagonal lattice $A_{2}$, with Gram matrix $[1,1 / 2 ; 1 / 2,1]$ (see [11]). In fact we can prove a stronger result:

Theorem 1: Given any two-dimensional lattice $L$, there exists a sequence of vectors $\boldsymbol{a}_{k}=\left(1, b_{k}, c_{k}\right) \in \mathbb{Z}^{3}$ such that the projected lattice $\Lambda_{\boldsymbol{a}_{k}}$ converges to a lattice that is geometrically similar to $L$.

Proof: $\quad$ Let $L$ have Gram matrix $[1, P ; P, Q]$ with $0 \leq$ $P \leq 1 / 2, Q \geq 1$. Using continued fraction approximations, choose an infinite sequence of positive integers $b_{k}$ such that $\sqrt{\Delta} b_{k}=n_{k}-\phi$, where $\Delta=Q-P^{2}, n_{k} \in \mathbb{Z}, \phi>0$ and $\phi \rightarrow 0$. Set $c_{k}=\left\lfloor\left(b_{k}+1 / b_{k}\right)\left(P+n_{k}+1\right)\right\rfloor$. The Gram matrix 
for $\Lambda_{a_{k}}$ is then

$$
\left(\begin{array}{cc}
1 & b_{k} c_{k} /\left(b_{k}^{2}+1\right) \\
b_{k} c_{k} /\left(b_{k}^{2}+1\right) & \left(c_{k}^{2}+1\right) /\left(b_{k}^{2}+1\right)
\end{array}\right) .
$$

The Minkowswki-reduced Gram matrix has the form [1, $P$ $\left.\epsilon_{1} ; P-\epsilon_{1}, Q+\epsilon_{2}\right]$, where $\epsilon_{1}, \epsilon_{2} \geq 0, \epsilon_{1}, \epsilon_{2} \rightarrow 0$.

For $n=4$, the target lattice is the face-centered cubic lattice $D_{3}$, which has Gram matrix

$$
\boldsymbol{G}_{3}=\left(\begin{array}{ccc}
1 & 0 & 1 / 2 \\
0 & 1 & 1 / 2 \\
1 / 2 & 1 / 2 & 1
\end{array}\right)
$$

Theorem 2: Let $\boldsymbol{a}_{k}=\left(1, b_{k}, c_{k}, d_{k}\right)$ where $b_{k}=2 k^{2}-k+$ $1, c_{k}=2 k^{2}+k+1, d_{k}=k\left(4 k^{2}+3\right)$. Then for $k \rightarrow \infty$, the projected lattice $\Lambda_{\boldsymbol{a}_{k}}$ converges to a lattice that is geometrically similar to $D_{3}$.

Proof: The lattice $\Lambda_{\boldsymbol{a}_{k}}$ has Gram matrix

$$
\boldsymbol{G}=\left(\begin{array}{ccc}
M-b_{k}^{2} & -b_{k} c_{k} & -b_{k} d_{k} \\
-b_{k} c_{k} & M-c_{k}^{2} & -c_{k} d_{k} \\
-b_{k} d_{k} & -c_{k} d_{k} & M-d_{k}^{2}
\end{array}\right),
$$

and determinant $M^{2}$, where $M=\left(k^{2}+1\right)\left(4 k^{2}+1\right)\left(4 k^{2}+3\right)$. A Minkowski-reduced Gram matrix is $\boldsymbol{G}_{k}=\boldsymbol{C}_{k} \boldsymbol{G} \boldsymbol{C}_{k}^{t r}$, where

$$
\begin{gathered}
\boldsymbol{C}_{k}=\left(\begin{array}{ccc}
k-1 & k & k(2 k-1) \\
k & k+1 & k(2 k+1) \\
0 & 0 & 1
\end{array}\right), \\
\boldsymbol{G}_{k}=\left(\begin{array}{ccc}
8 k^{4}+9 k^{2}+2 & \left(2 k^{2}+1\right)^{2} & -4 k^{4}-3 k^{2} \\
\left(2 k^{2}+1\right)^{2} & 8 k^{4}+9 k^{2}+2 & -4 k^{4}-3 k^{2} \\
-4 k^{4}-3 k^{2} & -4 k^{4}-3 k^{2} & 8 k^{4}+10 k^{2}+3
\end{array}\right) .
\end{gathered}
$$

This matrix is in Minkowski-reduced form [2], and $\mu(i)=$ $8 i^{4}+9 i^{2}+2$ is the smallest diagonal entry. As $k \rightarrow \infty$, the center density converges to $1 / \sqrt{32}$, the center density of $D_{3}$.

\section{An Achievable Bound}

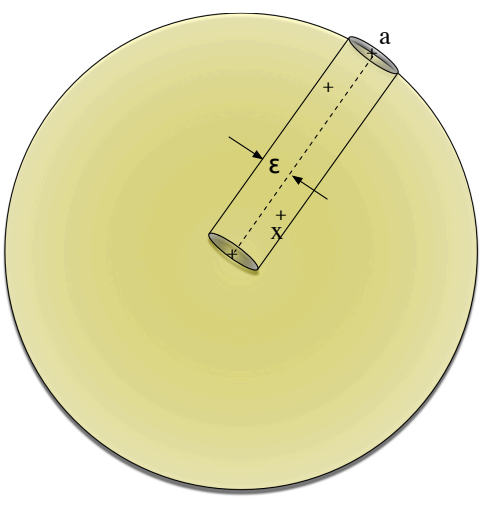

Fig. 4. A cylinder of radius $\epsilon$ anchored by the origin and a lattice point $\boldsymbol{a}$. The objective is to obtain an estimate for the average number of primitive lattice points in the cylinder, when we average over all primitive points $\boldsymbol{a}$ on a sphere of given radius.

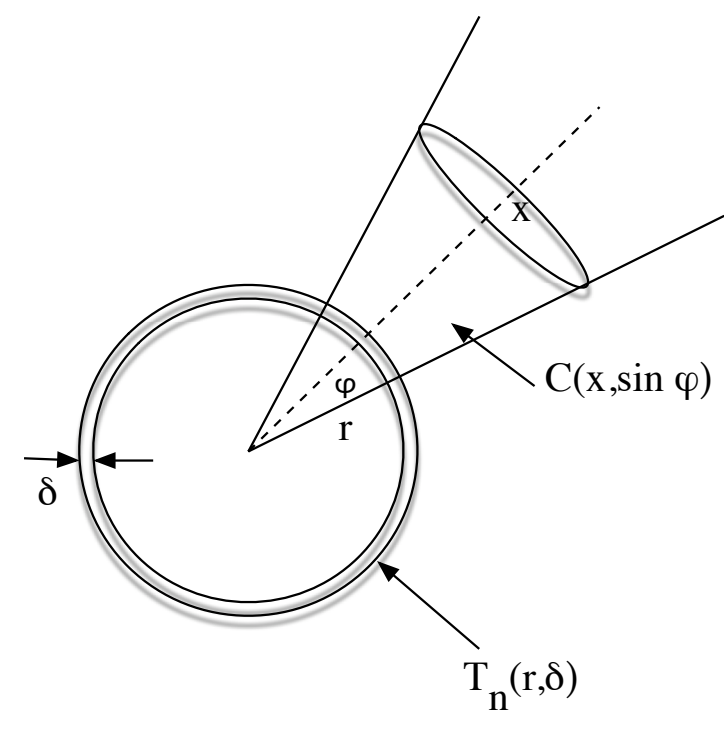

Fig. 5. Shell and cone in $\mathbb{R}^{n}$.

For general dimensions we can give a nonconstructive lower bound for the density that can be achieved by the projected lattice, using an averaging argument. Averaging over struts of constant volume is complicated by the fact that as the struts lengthen they become thinner, which makes it difficult to obtain accurate estimates of the number of points in the interior. This difficulty is overcome by summing over spherical caps whose volume grows with the length of the strut.

\section{A. Notation}

A vector $\boldsymbol{x}=\left(x_{1}, x_{2}, \ldots, x_{n}\right) \in \mathbb{Z}^{n}$ is is said to be primitive if $\operatorname{gcd}\left(x_{1}, x_{2}, \ldots, x_{n}\right)=1$. Let $B_{n}(r)=\{\boldsymbol{x} \in$ $\left.\mathbb{R}^{n},\|x\|<r\right\}$ be an open ball of radius $\mathrm{r}$ with center at the origin. The unit ball $B_{n}(1)$ will be abbreviated to $B_{n}$. Let $S_{n}(r)=\left\{\boldsymbol{x} \in \mathbb{R}^{n},\|\boldsymbol{x}\|=r\right\}$ be a sphere in $\mathbb{R}^{n}$ with radius $r$. Let $T_{n}(r, \delta)=\left\{\boldsymbol{x} \in \mathbb{R}^{n}: r(1-\delta)<\|\boldsymbol{x}\| \leq r\right\}$ be a shell of radius $r$ and thickness $r \delta$. Let $C_{n}(\boldsymbol{x}, \sin \phi)=$ $\left\{\boldsymbol{y} \in \mathbb{R}^{n}:\langle\boldsymbol{y}, \boldsymbol{x}\rangle \geq\|\boldsymbol{x}\|\|\boldsymbol{y}\| \sqrt{1-\sin ^{2} \phi}\right\}$ be a cone with axis $x$ and central angle $\phi \leq \pi / 2$. The intersection of a cone with a shell is called a spherical cap $S C_{n}(\boldsymbol{x}, \sin \phi, r, \delta):=$ $C_{n}(\boldsymbol{x}, \sin \phi) \bigcap T_{n}(r, \delta)$. The 'thin' shell $C_{n}(x, \sin \phi) \bigcap S_{n}(r)$ is abbreviated to $S C_{n}(x, \sin \phi, r)$.

\section{B. Lemmas}

Lemma 1: Let $\boldsymbol{x}, \boldsymbol{y} \in \mathbb{R}^{n}$ and let $t, s \in R$. Given $\epsilon>0, \epsilon \in$ $\mathbb{R}$, the following are equivalent:

$$
\begin{aligned}
& \text { (i) } \min _{t}\|\boldsymbol{x}-t \boldsymbol{y}\|<\epsilon \text {. } \\
& \text { (ii) } \min _{s}\|\boldsymbol{y}-s \boldsymbol{x}\|<\epsilon \frac{\|\boldsymbol{y}\|}{\|\boldsymbol{x}\|} \text {. }
\end{aligned}
$$

Proof: Let $t_{*}=\langle\boldsymbol{x}, \boldsymbol{y}\rangle /\|\boldsymbol{y}\|^{2}$ be the minimizer in (i) and let $s_{*}=\langle\boldsymbol{x}, \boldsymbol{y}\rangle /\|\boldsymbol{x}\|^{2}$ be the minimizer in (ii). Then $\left\|\boldsymbol{x}-t_{*} \boldsymbol{y}\right\|^{2}=\|\boldsymbol{x}\|-t_{*}^{2}\|\boldsymbol{y}\|^{2}=\|\boldsymbol{x}\|^{2}-\langle\boldsymbol{x}, \boldsymbol{y}\rangle^{2} /\|\boldsymbol{y}\|^{2}=$ $\left(\|\boldsymbol{x}\|^{2} /\|\boldsymbol{y}\|^{2}\right)\left(\|\boldsymbol{y}\|^{2}-s_{*}^{2}\|\boldsymbol{x}\|^{2}\right)=\left(\|\boldsymbol{x}\|^{2} /\|\boldsymbol{y}\|^{2}\right)\left\|\boldsymbol{y}-s_{*} \boldsymbol{x}\right\|^{2}<\epsilon^{2}$. The claim follows directly. 
The volume of the unit ball $B_{n}$ is given by $V_{n}=$ $\pi^{n / 2} / \Gamma\left(\frac{n}{2}+1\right)$, and the "area" (or $(n-1)$-dimensional measure) of the sphere $S_{n}$ is $A_{n}=n V_{n}$. The area of the spherical cap $S C_{n}(\boldsymbol{y}, \sin \phi, 1)$ is

$$
A_{n}(\phi)=(n-1) V_{n-1} \int_{0}^{\phi} \sin ^{n-2} \theta d \theta .
$$

We will need bounds on $A_{n}$ which are of the form

$$
u_{n} \sin ^{n-1} \phi \leq A_{n}(\phi) \leq U_{n} \sin ^{n-1} \phi .
$$

Specific values for $u_{n}$ and $U_{n}$ are given below. The following is well-known,

Lemma 2: The area of a spherical cap satisfies

$$
V_{n-1} \sin ^{n-1} \phi \leq A_{n}(\phi) \leq(1 / 2) n V_{n} \sin ^{n-1} \phi .
$$

Proof: The upper bound is the area of a hemisphere of radius $\sin \phi$ in $\mathbb{R}^{n}$. The lower bound is the volume of a sphere of radius $\sin \phi$ in $\mathbb{R}^{n-1}$.

Stirling's formula shows that the ratio of the upper bound to the lower bound is approximately $\sqrt{\pi n / 2}$.

Given $\boldsymbol{a} \in \mathbb{Z}^{n}$, let $\mathcal{L}_{\boldsymbol{a}}$ be the line $\{$ ta,$t \in \mathbb{R}\}$. If $\boldsymbol{a} \in$ $\mathbb{Z}^{n}$ is primitive, the only integer vectors in $\mathcal{L}_{\boldsymbol{a}}$ are integer multiples of $\boldsymbol{a}$. A vector $\boldsymbol{x} \in \mathbb{Z}^{n}$, not an integer multiple of $\boldsymbol{a}$, is said to be a nearest neighbor of $\mathcal{L}_{\boldsymbol{a}}$ if $d\left(\boldsymbol{x}, \mathcal{L}_{\boldsymbol{a}}\right):=\min _{t} \| \boldsymbol{x}-$ $t \boldsymbol{a} \| \leq d\left(\boldsymbol{x}^{\prime}, \mathcal{L}_{\boldsymbol{a}}\right)$ for any $\boldsymbol{x}^{\prime} \in \mathbb{Z}^{n}$ which is not a multiple of $\boldsymbol{a}$. If $\boldsymbol{x}$ is a nearest neighbor, then $d\left(\boldsymbol{x}, \mathcal{L}_{\boldsymbol{a}}\right) \leq 1$, since $d\left((0,0, \ldots, 1), \mathcal{L}_{\boldsymbol{a}}\right) \leq 1$. Note also that $d\left(\boldsymbol{x}, \mathcal{L}_{\boldsymbol{a}}\right)=d(\boldsymbol{x}+$ $\left.k a, \mathcal{L}_{\boldsymbol{a}}\right)$ for any $k \in \mathbb{Z}$ : nearest neighbors are not unique.

Lemma 3: Let $\boldsymbol{x}=\left(x_{1}, x_{2}, \ldots, x_{n}\right)$ be a nearest neighbor of $\mathcal{L}_{\boldsymbol{a}}$. Then $x$ is primitive.

Proof: Suppose that the $x$ is not primitive and that $p>$ 1 is a common divisor of its entries. The minimal distance between $\boldsymbol{x}$ and $\mathcal{L}_{\boldsymbol{a}}$ is $\|\boldsymbol{x}\| \sin \theta$, where $\theta$ is the angle $\theta$ between $\boldsymbol{x}$ and $\boldsymbol{a}$. But $\boldsymbol{x} / p$ is an integer vector and the angle between $\boldsymbol{x} / p$ and $\boldsymbol{a}$ is also $\theta$, which implies that $\boldsymbol{x} / p$ is strictly closer to the line $\mathcal{L}_{\boldsymbol{a}}$ than $\boldsymbol{x}$.

Lemma 4: The line $\mathcal{L}_{\boldsymbol{a}}$ has a nearest neighbor $\boldsymbol{x}$ such that $\|\boldsymbol{x}\|^{2} \leq\|\boldsymbol{a}\|^{2} / 4+1$.

Proof: If $\boldsymbol{x}$ is a nearest neighbor then so is $\boldsymbol{a}-\boldsymbol{x}$. Without loss of generality, assume that $\|x\|^{2} \leq\|\boldsymbol{a}-\boldsymbol{x}\|^{2}$. Equivalently $\langle\boldsymbol{a}, \boldsymbol{x}\rangle \leq\|\boldsymbol{a}\|^{2} / 2$. But $\min _{t}\|\boldsymbol{x}-t \boldsymbol{a}\|^{2}=\|\boldsymbol{x}\|^{2}-\langle\boldsymbol{x}, \boldsymbol{a}\rangle^{2} /\|\boldsymbol{a}\|^{2} \leq$ 1. Thus $\|\boldsymbol{x}\|^{2} \leq\|\boldsymbol{a}\|^{2} / 4+1$.

This lemma allows us to restrict the search for a nearest neighbor of $\mathcal{L}_{\boldsymbol{a}}$ to a ball of radius $\sqrt{1+\|\boldsymbol{a}\|^{2} / 4}$ centered at the origin.

\section{Derivation of the Achievable Bound}

Given $\epsilon>0$ and $\boldsymbol{a} \in \mathbb{Z}^{n}$, let $N(\boldsymbol{a}, \epsilon)$ be the number of primitive vectors $x$ in $\mathbb{Z}^{n}$ with length at most $\sqrt{1+\|\boldsymbol{a}\|^{2} / 4}$ and $d\left(\boldsymbol{x}, \mathcal{L}_{\boldsymbol{a}}\right)<\epsilon$. Let $\bar{N}(\epsilon, r, \delta)$ be the average of $N(\boldsymbol{a}, \epsilon)$ calculated with a uniform distribution over primitive vectors $\boldsymbol{a}$ in $T_{n}(r, \delta)$. If $\bar{N}(\epsilon, r, \delta)<1$ then there exists primitive $\boldsymbol{a}$,

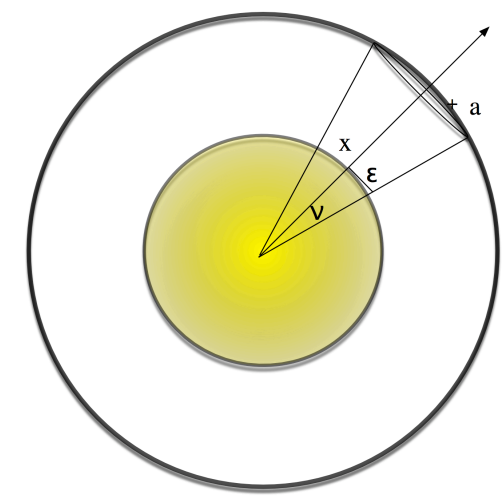

Fig. 6. For a given $\boldsymbol{x}$ on a sphere of radius $\nu$, any point $\boldsymbol{a}$ on a sphere of radius $r$ that lies inside the cone $C(x, \epsilon / \nu)$ will have $\boldsymbol{x}$ as an $\epsilon$-neighbor.

$\|\boldsymbol{a}\| \in(r(1-\delta), r]$ such that $N(\boldsymbol{a}, \epsilon)=0$. In other words, if $\bar{N}(\epsilon, r, \delta)<1$ a strut exists with length $\leq r$ and radius $\epsilon$.

Let $S_{n}^{\#}(k)=\left\{\boldsymbol{x} \in \mathbb{Z}^{n},\|\boldsymbol{x}\|^{2}=k\right\}$ be the $k$ th shell of $\mathbb{Z}^{n}$. Let $N_{n}(k)=\left|S_{n}^{\#}(k)\right|$ denote the number of points on the $k$ th shell of $\mathbb{Z}^{n}$. Let $M_{n}(r, \delta)$ be the number of primitive vectors $\boldsymbol{a}$ in the shell $T_{n}(r, r \delta)$. It is assumed that $\boldsymbol{a}$ is primitive in the following steps for calculating $\bar{N}(\epsilon, r, \delta)$.

$$
\begin{aligned}
& M_{n}(r, \delta) \bar{N}(\epsilon, r, \delta) \\
& \leq \sum_{\boldsymbol{a} \in T_{n}(r, \delta)} \sum_{\boldsymbol{x} \in B_{n}\left(\sqrt{\frac{r^{2}}{4}+1}\right), d\left(\boldsymbol{x}, \mathcal{L}_{\boldsymbol{a}}\right)<\epsilon} \sum_{\boldsymbol{x} \in B_{n}\left(\sqrt{\frac{r^{2}}{4}+1}\right)} 1 \\
& =\sum_{k=1} \sum_{\boldsymbol{a} \in T_{n}(r, \delta), d\left(\boldsymbol{a}, \mathcal{L}_{\boldsymbol{x}}\right)<\epsilon\|\boldsymbol{a}\|} 1 \\
& \sum_{\boldsymbol{x} \in S_{n}^{\#}(k)} 1,
\end{aligned}
$$

where we have exchanged the order of summation so as to count the points in the shadow (the number of points in a spherical cap) of a point $\boldsymbol{x}$ on shell $S_{n}^{\#}(k)$ and the inequality in the first step is because we count $x$ even if it not primitive. For a point $x \in S_{n}^{\#}(k)$, the number of primitive points in the shadow is given by

$$
\begin{aligned}
& \sum_{\boldsymbol{a} \in S C(\boldsymbol{x}, \epsilon / \sqrt{k}, r, \delta)} 1 \\
= & A_{n}\left(\frac{\epsilon}{\sqrt{k}}\right) \frac{1}{\zeta(n)} \int_{r(1-\delta)}^{r} x^{n-1} d x\left(1+o_{r}(1)\right) \\
= & A_{n}\left(\frac{\epsilon}{\sqrt{k}}\right) \frac{r^{n}}{n \zeta(n)}\left(1-(1-\delta)^{n}\right)\left(1+o_{r}(1)\right) \\
\leq & U_{n} \frac{\epsilon^{n-1}}{k^{(n-1) / 2}} \frac{r^{n}}{n \zeta(n)}\left(1-(1-\delta)^{n}\right)\left(1+o_{r}(1)\right)
\end{aligned}
$$

where $\lim _{r \rightarrow \infty} o_{r}(1)=0$, and we have used the inversion formula, [5], Thm. 268, to estimate the number of primitive points in $S C(\boldsymbol{x}, \epsilon / \sqrt{k}, r, \delta)$. Eq. (9) was used in the last step. 
Thus

$$
\begin{aligned}
& M_{n}(r, \delta) \bar{N}(\epsilon, r, \delta) \\
& \quad \leq \sum_{k=1}^{\frac{r^{2}}{4}+1} N_{n}(k) U_{n} \frac{\epsilon^{n-1}}{k^{\frac{n-1}{2}}} \frac{r^{n}}{n \zeta(n)}\left(1-(1-\delta)^{n}\right)\left(1+o_{r}(1)\right) .
\end{aligned}
$$

But

$$
M_{n}(r, \delta)=V_{n} \frac{r^{n}}{\zeta(n)}\left(1-(1-\delta)^{n}\right)\left(1+o_{r}(1)\right) .
$$

Thus

$$
\bar{N}(\epsilon, r, \delta) \leq\left(\sum_{k=1}^{\frac{r^{2}}{4}+1} \frac{N_{n}(k)}{n V_{n} k^{\frac{n-1}{2}}}\right) U_{n} \epsilon^{n-1}\left(1+o_{r}(1)\right) .
$$

Using summation by parts it can be shown that

$$
\lim _{m \rightarrow \infty} \sum_{k=1}^{m} \frac{N_{n}(k)}{n V_{n} k^{\frac{n-1}{2}}}=\sqrt{m} \text {. }
$$

Thus

$$
\bar{N}(\epsilon, r, \delta) \leq U_{n} \epsilon^{n-1} \frac{r}{2}\left(1+o_{r}(1)\right) .
$$

Since a strut of length $r$ and radius $\epsilon$ exists if $\bar{N}(\epsilon, r, \delta)<1$ we have established:

Theorem 3: Given $n>1, \delta_{2}>0$, there is a number $r_{0}\left(n, \delta_{2}\right)>0$ such that for all $r>r_{0}\left(n, \delta_{2}\right)$, there exists a primitive vector $\boldsymbol{a} \in \mathbb{Z}^{n}$ with $\|\boldsymbol{a}\|=r$ and a corresponding strut of radius $\epsilon$ satisfying

$$
\epsilon^{n-1}>\frac{2\left(1-\delta_{2}\right)}{r U_{n}}
$$

Proof: For given $\delta_{2}$ and $r$, let $\bar{\epsilon}$ satisfy $U_{n} \bar{\epsilon}^{n-1} r / 2=1-$ $\delta_{2}$. From 17 we know that the average quantity $\bar{N}(\bar{\epsilon}, r, \delta) \leq$ $U_{n} \bar{\epsilon}^{n-1}(r / 2)\left(1+o_{r}(1)\right)=\left(1-\delta_{2}\right)\left(1+o_{r}(1)\right)=\left(1-\delta_{2}\right)+$ $\left(1-\delta_{2}\right) o_{r}(1)$. Pick $r_{0}\left(n, \delta_{2}\right)$ such that $\left|\left(1-\delta_{2}\right) o_{r}(1)\right|<\delta_{2} / 2$ for $r>r_{0}\left(n, \delta_{2}\right)$. Thus for $r>r_{0}\left(n, \delta_{2}\right), \bar{N}(\bar{\epsilon}, r, \delta) \leq$ $U_{n} \bar{\epsilon}^{n-1}(r / 2)\left(1+o_{r}(1)\right) \leq 1-\delta_{2} / 2<1$. This implies that for any $r>r_{0}\left(n, \delta_{2}\right)$ there exists primitive $\boldsymbol{a}$ with $\|\boldsymbol{a}\|=r$ and a corresponding strut of radius $\epsilon>\bar{\epsilon}$ for which (18) is satisfied.

Let us compare this with the Minkowski-Hlawka result. If the strut associated with the vector $\boldsymbol{a}$ has radius $\epsilon$, the packing density of the $(n-1)$-dimensional lattice $\Lambda_{\boldsymbol{a}}$ obtained by projecting $\mathbb{Z}^{n}$ onto $\boldsymbol{a}^{\perp}$ is $\Delta_{n-1}=(\epsilon / 2)^{n-1} V_{n-1}\|\boldsymbol{a}\|$. The result of Theorem 3 implies that lattices with packing density

$$
\Delta_{n-1}=\frac{V_{n-1}}{2^{n-1} U_{n}}
$$

can be obtained by projecting $\mathbb{Z}^{n}$ into a subspace orthogonal to a primitive vector in $\mathbb{Z}^{n}$ whose norm is suitably large. The bound stated in Lemma 2 implies $U_{n} \leq n V_{n} / 2$. Thus

$$
\Delta_{n-1} \geq \frac{V_{n-1}}{n 2^{n-2} V_{n}} \sim \frac{1}{\sqrt{2 \pi n} 2^{n-2}}
$$

is achievable, compared with $\frac{\zeta(n-1)}{2^{n-2}}$ from the MinkowskiHlawka bound (2) for the packing density achievable by unrestricted lattices. Our bound is worse by a factor of $\sqrt{2 \pi n}$.

\section{SUMMARY}

The fat strut problem is posed, motivated by a question in communication theory. It is shown that finding a good solution is equivalent to finding a vector in the integer lattice $\mathbb{Z}^{n}$ with the property that the lattice obtained by projecting $\mathbb{Z}^{n}$ into the orthogonal complement of the vector has large packing density. Constructions are given for $n=3,4$ and an achievable bound is derived by an averaging argument.

\section{REFERENCES}

[1] E. Biglieri and M. Elia. Cyclic group codes for the Gaussian channel. IEEE Trans. Inform. Theory, 22:624-629, Sept. 1976.

[2] J. W. S. Cassels. An Introduction to the Geometry of Numbers. SpringerVerlag, 1971.

[3] J. H. Conway and N. J. A. Sloane. Sphere Packings, Lattices and Groups. Springer-Verlag, New York, 3rd ed., 1993.

[4] P. M. Gruber and C. G. Lekkerkerker. Geometry of Numbers. NorthHolland, Amsterdam, 2nd ed., 1987.

[5] G. H. Hardy and E. M. Wright. An Introduction to the Theory of Numbers. Oxford University Press, New York, 5th ed., 1979.

[6] A. Heppes. Ein satz über gitterförmige kugelpackungen. Ann. Univ. Sci. Budapest. Eötvös Sect. Math., 3-4:89-90, 1960/1961.

[7] E. Hlawka. Zur geometrie der zahlen. Math. Zietschr, 49:285-312, 1943.

[8] J. Horváth. Über die durchsichtigkeit gitterförmiger kugelpackungen. Studia Sci. Math. Hungar, 5:421-426, 1970.

[9] C. E. Shannon. Communication in the presence of noise. Proc. IRE, 37:30-41, Jan. 1949.

[10] V. A. Vaishampayan and S. I. R. Costa. Curves on a sphere, shiftmap dynamics, and error control for continuous alphabet sources. IEEE Trans. Inform. Theory, 49(7):1658-1672, July 2003.

[11] V. A. Vaishampayan, N. J. A. Sloane and S. I. R. Costa. Dynamical systems, curves and coding for continuous alphabet sources. In Proceedings, 2002 Information Theory Workshop, pages 111-114, October 2002. 